\title{
Conformal Deformation of Equilibrium Measures in Normal Random Ensembles
}

\author{
A.M. Veneziani ${ }^{\dagger}$ \\ E-mail: alexei.veneziani@ufabc.edu.br \\ T. Pereira ${ }^{\dagger}$ \\ E-mail: tiago.pereira@ufabc.edu.br
}

\section{D.H.U. Marchetti*}

E-mail: marchetti@if.usp.br

† Centro de Matemática, Computação e Cognição

Universidade Federal do ABC, Santo André, Brasil

*Instituto de Física, Universidade de São Paulo, São Paulo, Brasil

\begin{abstract}
We investigate the eigenvalues statistics of ensembles of normal random matrices when their order $N$ tends to infinite. In the model, the eigenvalues have uniform density within a region determined by a simple analytic polynomial curve. We study the conformal deformations of equilibrium measures of normal random ensembles to the real line and give sufficient conditions for it to weakly converge to be a Wigner measure.
\end{abstract}

\section{Introduction and Statement of Results}

Since early fifties Hermitian random matrix theory plays an important role in the statistical description of the spectra of complex systems $[1,2]$. Recently, nonHermitian random matrices have been used to treat problems in superconductor physics with columnar defects [3, 4], in quantum chaotic systems [5], and in quantum chromodynamics $[6,7]$. Normal random matrix ensembles have been playing a major role in several areas such as in the study of fractional quantum Hall effect [8], quantum Hele-Shaw flows [9], integrable hierarchies [10], and integrable structure of the Dirichlet boundary problem $[11,12]$.

The interplay between non-Hermitian and Hermitian random matrices has attracted a great deal of attention [7, 13, 14]. Recent investigations consider matrix ensembles of the form $J=H-i \Gamma$, subjected to the so called weakly non-Hermicity condition: $\lim _{N \rightarrow \infty} N \operatorname{Tr} \Gamma^{2} / \operatorname{Tr} H^{2}<\infty$. These ensembles appear, for example, in the description of quantum scattering in systems with chaotic dynamics and serve to describe resonance 
statistics. If $H$ and $\Gamma$ are independently sampled from Gaussian unitary ensembles, the eigenvalues of $J$ has, for large $N$, the same statistics of the eigenvalues of a Hermitian ensemble (see [7] for a review of this and related results). The continuous behavior of the eigenvalue statistics of weakly non-Hermitian ensemble has motivated the present investigation.

We are interested in the equilibrium measure (i.e., the $N \rightarrow \infty$ limit of the $1-$ point function) of non-Hermitian ensemble whose support is closed to the real line. We consider ensembles of normal matrices whose eigenvalues have uniform density within a domain in the complex plane. We give conditions on the random matrix ensemble under which the corresponding equilibrium measure behaves continuously when its support collapses to a segment of the real line. Under additional restrictions, the limiting measure is shown to satisfy the Wigner semi-circular law.

We address the problem by using the so-called invariant ensemble model, characterized by the probability of finding a $N \times N$ matrix $M$ of a class within the ensemble given by

$$
P(M) d M \propto \exp \{-N \operatorname{Tr}[V(M)]\} d M,
$$

with the trace $\operatorname{Tr}[V(M)]$ and the Riemann volume $d M$ invariant under unitary transformations. The corresponding eigenvalue density, in the limit $N \rightarrow \infty$, depends on the particular form of $V(M)$. For the Wigner ensemble $M$ is a Hermitian matrix whose entries are independent and identically distributed Gaussian random variables with zero mean and variance $\sigma^{2} / N$. The potential reads $V(M)=\frac{1}{\sigma^{2}} M^{*} M\left(M^{*}\right.$ is the Hermitian conjugate of $M)$. The density of eigenvalues follows the Wigner semi-circular law supported on $[-2 \sigma, 2 \sigma][2]$ :

$$
d \mu_{W}(x)=\frac{1}{2 \pi \sigma} \sqrt{4-x^{2} / \sigma^{2}} \chi_{[-2 \sigma, 2 \sigma]}(x) d x,
$$

where $\chi_{A}(x)=1$ if $x \in A$ and 0 otherwise.

A particularly interesting potential has been put forward by Wiegmann, Zabrodin, and coworkers $[10,11,12,15]$ who established a connection between normal random matrices and conformal mappings. They considered

$$
V(M)=\frac{1}{t_{0}}\left(M^{*} M-p(M)-p(M)^{*}\right)
$$

where

$$
p(z)=\sum_{j \geq 1} t_{j} z^{j}
$$

with $t_{0}>0$ and $t_{j} \in \mathbb{C}$. As $N \rightarrow \infty$, they showed, at the level of formal manipulations, that : $(A)$ the density of eigenvalues is uniform within a simply connected domain $D \subset \mathbb{C}$ whose boundary is given by a simple analytic curve $\gamma ;(B)$ the domain $D$ is characterized by the fact that its exterior harmonic moments

$$
t_{j}=\frac{1}{2 \pi i j} \oint_{\gamma} \bar{z} z^{-j} d z, \quad j \geq 1
$$


where $\pi t_{0}$ stands for the area of $D$, are the coefficients of $(4)$; and $(C)$ the Riemann mapping from the exterior of the unit disk onto the exterior of the domain $D$ obeys, as a function of the $t_{j}$, the equations of the integrable dispersionless Toda hierarchy.

Potentials of the form (3) give rise two sorts of mathematical problems. Except in the case of polynomial $p(z)$ of degree 2 , where the domain $D$ is bounded by an ellipse, $V(z)$ is not bounded from bellow and integrals with respect to (1) diverge. The other problem concerns with the fact that $D$ may not be uniquely determined by the moments (5). From the point of view of equilibrium measures (see Section 2), a relevant fraction of eigenvalues of a $M$ may escape to infinity or to another Riemann surface.

Recently, the results $(A)$ and $(B)$ have been set in a rigorous frame by Elbau and Felder [16]. To avoid the former problems, they consider the following restrictions:

\section{Elbau-Felder Potential. If}

$$
p(z)=t_{1} z+t_{2} z^{2}+\cdots+t_{n+1} z^{n+1}
$$

is an analytic polynomial of degree $n+1$ with $t_{0}>0$ and $\boldsymbol{t}=\left(t_{1}, \ldots, t_{n+1}\right) \in \mathbb{C}^{n+1}$ such that $t_{1}=0,\left|t_{2}\right|<1 / 2$, Elbau-Felder potential is a real-valued function on $\mathbb{C}$ given by

$$
V(z)=\frac{1}{t_{0}}\left(|z|^{2}-p(z)-\overline{p(z)}\right) \text {. }
$$

It can be shown by direct computations that $V(z)$, under the above conditions, is positive in a neighborhood of $z=0$ and has a non-degenerate absolute minimum at $z=0$. From now on, $V(z)$ shall stand for the Elbau-Felder potential. The problem of divergence of the integrals is solved in a naïve way - imposing that the eigenvalues of matrices within the normal ensemble remains bounded:

Elbau-Felder Ensemble. Let $\Sigma \subset \mathbb{C}$ be the closure of a bounded open set that contains the origin and consider the following class of matrices

$$
\mathcal{N}_{N}(\Sigma)=\left\{A \in \operatorname{Mat}_{\mathbb{C}}(N):\left[A, A^{*}\right]=0, \sigma(A) \subset \Sigma\right\}
$$

where $\sigma(A)$ denotes the spectrum of $A$. An ensemble is said to be of Elbau-Felder type of degree $n+1$ if it fulfills conditions stated between (6) and (8).

A closed polynomial curve $\gamma$ of degree $n$ can be parameterized by

$$
w \mapsto h(w)=r w+\sum_{j=0}^{n} a_{j} w^{-j}, \quad|w|=1
$$

for some $r>0$ and the $a_{j} \in \mathbb{C}$. Elbau and Felder have shown that, as long as $\left|t_{2}\right|<1 / 2$ and $t_{0}$ is small enough, the problem of determining the exterior moments $t_{j}$ out of the curve has a unique solution for simple closed analytic polynomial curves. Introducing $\rho=r^{2}$ and $\alpha_{j}=a_{j} / r^{j}$, they give a set of equations that defines an invertible map $F:\left(\rho, \alpha_{0}, \ldots, \alpha_{n}\right) \longrightarrow\left(t_{0}, \ldots, t_{n+1}\right)$ from $\mathbb{R} \times \mathbb{C}^{n+1}$ into itself about $\left(0,0,2 \bar{t}_{2}, \ldots,(n+1) \bar{t}_{n+1}\right)\left(\bar{t}_{j}\right.$ stand for the complex conjugate of the $\left.t_{j}\right)$. The eigenvalue density is then shown to be uniform in $D$ by the Euler-Lagrange variational equations. The parameter $r$ is essential for these results since it controls the smoothness of the 
curve $\gamma$, which looks like ellipses as $r$ shrinks to zero. We refer to Theorem 2 for a precise statement.

In the present work, we study conformal deformations of the equilibrium measures of Elbau-Felder ensembles, which are subjected to changes on the potential $V(z)$ through the parameters $\left(t_{0}, \boldsymbol{t}\right)=\left(t_{0}, t_{1}, \ldots, t_{n+1}\right)$. This is achieved by considering a family of polynomial curves of degree $n: w \mapsto h(w ; s)$, with the $a_{j}(s)$ depending on a parameter $s \in(0,1]$. This family is chosen so that $h\left(w ; s_{0}\right) \equiv h(w)$, for some $s_{0} \in(0,1]$, parameterizes the initial curve $\gamma$ whose interior domain $D$ supports the eigenvalues. After the construction of $h(w ; s)$, the support $D$ (resp. the external harmonic moments $t_{j}$ ) also depends on $s$ under $s \mapsto D(s)$ (resp. $t \mapsto t_{j}(s)$ ). We require, in addition, that the exterior domain $D^{-}(s)=\mathbb{C} \backslash D(s)$ tends, as $s$ goes to 0 , to a slit domain $\mathbb{C} \backslash[-2 r, 2 r]$ for some $r>0$. By equation (9), the polynomial curve which attains to this limit satisfies $\lim _{s \rightarrow 0} a_{1}(s)=r$ and $\lim _{s \rightarrow 0} a_{j}(s)=0$ for $j \neq 1$. In terms of the external harmonic moments, it satisfies $\lim _{s \rightarrow 0} t_{2}(s)=1 / 2$ and $\lim _{s \rightarrow 0} t_{j}(s)=0$ for $j \neq 2$. Since in this limit $t_{2}$ approaches $1 / 2$, the Elbau-Felder conditions will force $r$ to 0 and $\lim _{s \rightarrow 0} D^{-}(s)$ becomes a puncturated instead of a slit domain (see Remark 2 below).

We have two main problems. First, we want to establish a one-to-one and onto relation between the pair $\left(t_{0}, \boldsymbol{t}(s)\right)$, with $\boldsymbol{t}(s)=\left(t_{1}=0, t_{2}(s), \ldots, t_{n+1}(s)\right)$ the external harmonic moments of a (simple) curve $\gamma(s)$ and its parameterization by $h(w ; s)$, uniformly in $(0,1]$. The second problem concerns the behavior of the equilibrium measure under the deformation process. The existence and uniqueness of the equilibrium measure are granted only for some $s_{0}$ (by hypothesis) and the question is whether it remains valid for every $s \in\left(0, s_{0}\right]$. These problems are addressed in the theorem bellow.

To state our result, we denote by $\boldsymbol{\tau}=\left(\tau_{1}, \ldots, \tau_{n+1}\right)$ a vector on the affine space $Z \subset \mathbb{C}^{n+1}$ with $\tau_{1}=0$ and $\left|\tau_{2}\right|=1$ (note that $\tau_{2}=\exp \left\{i \arg \tau_{2}\right\}$ ) and consider, for $s \in(0,1]$, the following parameterization

$$
\begin{aligned}
& t_{1}(s)=0 \\
& t_{2}(s)=\frac{\sqrt{1-s}}{2} \exp \left(i s^{\Delta_{2}} \arg \tau_{2}\right) \\
& t_{j}(s)=s^{\Delta_{j}} \tau_{j} \quad \text { for all } \quad 3 \leq j \leq n+1
\end{aligned}
$$

with $\Delta_{j} \geq 1$. As long as $s>0$ (i. e., $\left|t_{2}\right|<1 / 2$ ), Theorem 2 of Elbau-Felder guarantees existence of a unique invertible map from $\left(\rho, \alpha_{0}, \ldots, \alpha_{n}\right)$ to $\left(t_{0}, t_{1}, \ldots, t_{n+1}\right)$, whose inverse defines $\rho=\rho\left(t_{0}, \boldsymbol{t}\right)$ and $\alpha_{j}=\alpha_{j}\left(t_{0}, \boldsymbol{t}\right)$, for sufficiently small $t_{0}$. We write $h\left(w ; t_{0}, \boldsymbol{t}\right)$ for the parameterization of a simple polynomial curve $\gamma$ whose external moments and the area of its interior domain $D$ are $\boldsymbol{t}=\left(t_{1}, \ldots, t_{n+1}\right)$ and $\pi t_{0}$. By the Elbau-Felder results, every interior domain $D$ of $\gamma$ supports the equilibrium measure provided the parameter $r$ is such that $r^{2}<\rho(\delta, \boldsymbol{t})$ for some $\delta>0$. So, for a given $\left(t_{0}, \boldsymbol{t}\right)$ with $t_{0}<\delta$, there exist $s_{0}$ and $\boldsymbol{\tau}$ such that $t_{j}=t_{j}\left(s_{0}\right)$ (with $\Delta_{j}$ fixed) and we write $h\left(w ; s_{0}, r, \boldsymbol{\tau}\right)$ for the parameterization $h\left(w ; t_{0}, \boldsymbol{t}\right)$ of the initial simple polynomial curve $\gamma$. Note that $r$ is uniquely defined by $\left(t_{0}, \boldsymbol{t}\right)$ and $\left|t_{2}\right|$ is determined by the value of $s_{0}$ : $\left|t_{2}\right|^{2}=\left(1-s_{0}\right) / 4$. That is, our parameterization takes into account all possible simple 
polynomial curve $\gamma$, whose interior domain $D$ supports the density of eigenvalues of the Elbau-Felder ensemble, as its initial condition. With this notation our main result reads

Theorem 1 Consider a one-parameter family of Elbau-Felder ensemble $s \mapsto$ $\left(t_{0}, \boldsymbol{t}(s)\right) \in \mathbb{R} \times \mathbb{C}^{n+1}$, with $t_{0}>0$ and $t_{j}(s)$, for $j \neq 0$ and each $s \in(0,1]$, satisfying (10). Then, there exist $s_{0} \in(0,1], \delta=\delta\left(s_{0}, \boldsymbol{\tau}\right)>0$ and, consequently, $r_{0}=r_{0}\left(s_{0}, \boldsymbol{\tau}\right)>0$ such that for every $s \in\left(0, s_{0}\right)$ and $0<r<r_{0}$ :

1) There is a unique simple analytic closed polynomial curve $\gamma=\gamma(s, r, \boldsymbol{\tau})$ of degree $n$, parameterized by $h(w ; s, r, \boldsymbol{\tau})$, with external harmonic moments $\boldsymbol{t}(s)$ and area of interior domain $\pi t_{0}$ where $t_{0}$, expressed in terms of $s, r$ and $\boldsymbol{\tau}$, is monotonic in $s$ and satisfies $0<t_{0}<\delta$ with $\lim _{s \rightarrow 0} t_{0}=0$.

2) The eigenvalue density is uniform within $D$, the interior domain of $\gamma$, for every $0<r<r_{0}$ and $s$ sufficiently small. Moreover, if $\Delta_{j}>1$ then the equilibrium measure can be conformally deformed, as s goes to 0, into a Wigner measure with support on $[-2 r, 2 r]$.

Remark 1 As long as $0<r<r_{0}, h(w ; s, r, \boldsymbol{\tau})=r w+\sum_{j=0}^{n} r^{j} \alpha_{j} w^{-j}$ is a Riemann mapping from the exterior of the unit disk onto the exterior domain $D$ of $\gamma$ and, as stated in item 1$)$, the area $\pi t_{0}$ of the domain $D$ remains positive for all $s \in\left(0, s_{0}\right)$.

Remark 2 The assumption $\left|t_{2}\right|<1 / 2$ in Theorem 2 breaks down when the exterior domain $D_{-}(s)=\mathbb{C} \backslash D(s)$ is deformed into the slit domain $\mathbb{C} \backslash[-2 r, 2 r]$. In Section 4 we generalize Elbau-Felder's results using Crandall-Rabinowitz bifurcation theory from simple eigenvalues (see e.g. [17]) to construct a parameterization that allow us to let $t_{2} \rightarrow 1 / 2$ maintaining the parameter $r$ away from 0 .

Remark 3 The Crandall-Rabinowitz theory allows us to obtain, by using the implicity function theorem, the leading order in $s$ of the $\alpha_{j}=\alpha_{j}(s, \boldsymbol{\tau})$. If $\Delta_{j}>1$ for $0 \leq j \leq n$, then, as $s \rightarrow 0$, we have (see Corollary 1)

(i) $\alpha_{0}(s)=o(1)$,

(ii) $\alpha_{1}(s)=1-s / 2+o(s)$,

(iii) $\alpha_{j}(s)=o(s)$ for $2 \leq j \leq n$.

This relation will be used to prove the second part of item 2) of Theorem 1.

Remark 4 Notice that the equilibrium measure completely characterizes the statistical behavior of the matrix ensemble. Hedenmalm and Makarov [9] proved that $k$-point correlation measure for Normal Random Matrix Ensembles in the limit $N \rightarrow \infty$ weakly converges to $k$ products of the equilibrium measure. Therefore, the conformal deformation can be extrapolated to the high order correlation functions associated with the Elbau-Felder ensemble. 
This paper is organized as follows. Section 2 presents some preliminary results and introduces the two ingredients, the balayage problem and the Schwarz function, required for the proof of the second part of item 2) of Theorem 1. In Section 3 we prove two auxiliary results, Propositions 1 and 2. Section 4 uses Crandall-Rabinowitz bifurcation theory from simple eigenvalues to solve the map $\left(t_{0}, \boldsymbol{t}\right)=F(\rho, \alpha)$ implicitly for $\alpha=\left(\alpha_{0}, \ldots, \alpha_{n}\right)$, in a neighborhood of $(\rho, \boldsymbol{\alpha})=\left(0, \boldsymbol{\alpha}^{*}\right)$ in $\mathbb{R} \times \mathbb{C}^{n+1}, \alpha_{j}^{*}=(j+1) \bar{t}_{j+1}$ with $t_{1}=0$ and $t_{2}=1 / 2$, as a function of $\left(\rho, t_{0}, \boldsymbol{\tau}\right)$. Theorem 4 in Section 5 gives an explicit expression of the Balayage measure for the potential $V$. Section 6 concludes the proof of Theorem 1 based in Lemma 1 and Section 7 gives some examples. We present in Section 8 our conclusions and Lemma 2 is proved in Appendix A.

\section{Basic Setting}

\subsection{Eigenvalue Distribution for Normal Ensembles}

For normal unitarily invariant ensembles, we can write Eq. (1) in terms of the spectral coordinates. The joint probability of the eigenvalues $\left\{z_{i}\right\}_{i=1}^{N}$ of $M$ reads

$$
P_{N}\left(z_{1}, \cdots, z_{N}\right) \propto \exp \left\{-\left(2 \sum_{1 \leq i<j \leq N} \log \left|z_{i}-z_{j}\right|^{-1}+N \sum_{i=1}^{N} V\left(z_{i}\right)\right)\right\} .
$$

Introducing the empirical measure of the eigenvalues

$$
d \mu_{N}(z)=N^{-1} \sum_{i=1}^{N} \delta\left(z-z_{i}\right) d^{2} z
$$

(11) can be written as

$$
P_{N}\left(z_{1}, \cdots, z_{N}\right)=Z_{N}^{-1} e^{-N^{2} I^{V}\left(\mu_{N}\right)},
$$

where $Z_{N}$ is the normalization and

$$
I^{V}(\mu) \equiv \int\left(V(z)+U^{\mu}(z)\right) d \mu(z)
$$

is the total energy. The logarithmic potential associated with $\mu$ given by

$$
U^{\mu}(z) \equiv \int \log |z-w|^{-1} d \mu(w)
$$

The integrals with respect to (11) have, in the limit $N \rightarrow \infty$, dominant contribution governed by a variational problem:

$$
E^{V} \equiv \inf _{\mu \in \mathcal{M}(\Sigma)} I^{V}(\mu)
$$

where the infimum is taken over the set $\mathcal{M}(\Sigma)$ of Borel probability measures in $\Sigma \subset \mathbb{C}$. If a probability measure $\mu^{V}$ satisfying

$$
E^{V}=I\left(\mu^{V}\right)
$$

exists, it is called the equilibrium measure associated with $V$. The empirical measure (12) is known to converge weakly to a unique equilibrium measure as $N \rightarrow \infty$ (see [2] for Hermitian ensembles and [9] for normal ensembles). 
Theorem 2 (Elbau-Felder) Consider the Elbau-Felder ensemble of degree $n$. There is $\delta>0$ such that for all $0<t_{0}<\delta$ a unique equilibrium measure $d \mu$ exists and is uniform within a domain $D \subset \Sigma$ that contains the origin:

$$
d \mu=\frac{1}{\pi t_{0}} \chi_{D}(z) d^{2} z
$$

$D$ is uniquely determined by the exterior harmonic moments $\left(t_{1}=0\right)$

$$
\begin{aligned}
\pi t_{0} & =\int_{D} d^{2} z \quad \text { the area of } D \\
t_{k} & =\frac{1}{2 \pi i k} \oint_{\gamma} \bar{z} z^{-k} d z, \text { if } k=2, \cdots, n+1, \\
t_{k} & =0 \text { if } k>n+1
\end{aligned}
$$

and its boundary $\gamma$ is a simple closed analytic polynomial curve of degree $n$; if $h(w)=$ $r w+a_{0}+a_{1} / w+\cdots+a_{n} / w^{n},|w|=1$, parameterizes $\gamma$, then

$$
t_{0}=r^{2}-\sum_{j=1}^{n} j\left|a_{j}\right|^{2} .
$$

There exist homogeneous universal polynomials $P_{j k} \in \mathbb{Z}\left[r, a_{0}, \cdots, a_{k-j}\right]$ of degree $k-j+1$, $1 \leq j \leq k \leq n+1$ such that

$$
j t_{j}=\bar{a}_{j-1} r^{-j+1}+\sum_{k=j}^{n} \bar{a}_{k} r^{-k} P_{j k}\left(r, a_{0}, \cdots, a_{k-j}\right)
$$

is an invertible transformation from $\mathbb{R} \times \mathbb{C}^{n}$ into itself in a neighborhood of $\left(r^{2}, a_{0}, a_{1} / r \ldots, a_{n} / r^{n}\right)=\left(0,0,2 \bar{t}_{2}, \ldots,(n+1) \bar{t}_{n+1}\right)$. For $r$ sufficiently small , the function $h(w)$ is a Riemann mapping from the exterior of the unit disk onto the exterior of $D$.

The proof of existence of the equilibrium measure requires to verify Euler-Lagrange type equations. Let

$$
E(z)=V(z)+\frac{2}{\pi t_{0}} \int_{D} \log \left|\frac{z}{\zeta}-1\right|^{-1} d^{2} \zeta
$$

be a function defined in $\Sigma$ given by $V$ plus the logarithmic potential (14) associated with the uniform measure $\mu$ in $D$. Lemma 6.3 of [16] shows that $E(z)=0$ holds for almost every $z \in D$. According to Corollary 3.5 of [16], $\mu$ is the unique equilibrium measure if

$$
E(z) \geq 0 \text { for every } z \in \Sigma \backslash D \text {. }
$$

We extend Elbau-Felder's proof to the near-slit-domains in Section 4. 


\subsection{Balayage Problem}

We tackle the problem of analyzing conformal deformations of the equilibrium measures by balayage techniques [18]. This allows us to solve the problem focusing only on the behavior of the boundary $\gamma$ of $D$. Therefore, in our approach the balayage technique plays a major role. Let $G \subset \overline{\mathbb{C}}$ be an open set and $\partial G$ its boundary.

Let $\nu$ be a probability measure on $G$ (such that $\nu(\overline{\mathbb{C}} \backslash G)=0$ ) and let the logarithmic potential $U^{\nu}$ (see Eq. (14)) be finite and continuous on $G$. The balayage problem (or "sweeping out" problem) consists in finding a probability measure $\widehat{\nu}$ with support on $\partial G$ such that

$$
U^{\nu}=U^{\widehat{\nu}} \text { almost everywhere on } \partial G \text {. }
$$

We call $\widehat{\nu}$ the balayage measure associated with $\nu$. Throughout this work, we consider the following space of functions:

Definition 1 Let $G \subset \mathbb{C}$ be a bounded open set. We denote by $\mathcal{H}(G)$ the space of all holomorphic functions on $G$ and continuous on its closure $\bar{G}$.

If $G \subset \mathbb{C}$ is a bounded open set and $\nu$ is a probability measure with compact support in $G$, then $\widehat{\nu}$ is the unique measure supported in $\partial G$ satisfying $(22)$ and such that $U^{\widehat{\nu}}(z)$ is bounded in $\partial G$. In addition, $\widehat{\nu}$ possesses the following property (see Theorem $I I-4.1$ of $[19])$ :

$$
\int_{G} f d \nu=\int_{\partial G} f d \widehat{\nu}
$$

holds for every $f \in \mathcal{H}(G)$.

We may choose $G$ the interior $\dot{D}=D \backslash \gamma$ of the compact support $D$ of the equilibrium measure $\mu$ associated with $V$. In this case, we write $\mu=\left.\mu\right|_{\dot{D}}+\left.\mu\right|_{\gamma}$ and sweep out only the part $\left.\mu\right|_{\dot{D}}$ lying on $G: \widehat{\mu}=\widehat{\left.\mu\right|_{\dot{D}}}+\left.\mu\right|_{\gamma}$. Since the equilibrium measure has no mass concentrated in $\gamma$, we have $\left.\mu\right|_{\gamma} \equiv 0$. The balayage measure associated with the equilibrium measure $\mu$ is denoted simply by $\widehat{\mu}$ and has support in $\gamma$.

\subsection{Parametric Curves and Schwarz Function}

The following definitions will be important for the characterization of the curves appearing in our main result. We shall start with the basic

Definition $2 A$ curve $\Gamma$ in $\mathbb{C}$ is said to be simple if there exist a parameterization $t \mapsto h(t)$ for $t \in[a, b]$ such that $h(t)$ is injective, $i$. e., if for all $x, y \in[a, b] h(x) \neq h(y)$ when $x \neq y$. If $h(a)=h(b)$, in this case $\Gamma$ is said to be a simple closed curve. A curve $\Gamma$ in $\mathbb{C}$ is said to be an analytic curve if there exist a parameterization $t \mapsto h(t)$ for $t \in[a, b]$ such that $h$ is analytic and $h^{\prime}(t) \neq 0$ for $t \in[a, b]$.

Next we introduce the polynomial curves on the complex plane. 
Definition 3 A curve $\Gamma$ in $\mathbb{C}$ is said to be a polynomial curve of degree $n$ if it is parametrically represented as

$$
h(w)=r w+a_{0}+a_{1} w^{-1}+\ldots+a_{n} w^{-n} .
$$

with $r>0, a_{n} \neq 0$ and $|w|=1$.

We shall define the Schwarz function

Definition 4 Let $\Gamma$ in $\mathbb{C}$ be an analytic arc and let $\Omega$ be a strip-like neighborhood of $\Gamma$. The Schwarz function $S$ is the unique analytic function on $\Omega$ such that

$$
S(z)=\bar{z}, \quad z \in \Gamma \text {. }
$$

For a treatise on the Schwarz function with applications see [20, 18].

Remark 5 Hereafter, $\gamma$ denotes a simple closed analytic polynomial curve. Moreover, $S$ stands for the Schwarz function of $\gamma$.

Schwarz function $S$ will play a major role in the conformal deformation of the Elbau-Felder ensemble. We shall show the balayage measure is proportional to the Schwarz function $S$.

\section{Riemann Map}

We shall prove some auxiliary results, which concern the behavior of the family $h(w ; s)$ as the $\gamma(s)$ is deformed to the real line.

Proposition 1 For $r>0$, let $a_{j}:(0,1] \rightarrow \mathbb{C}$, with $0 \leq j \leq n$, be continuous functions such that

$$
\xi(s):=r-\sum_{j=1}^{n} j\left|a_{j}(s)\right|>0
$$

holds for every $s \in(0,1]$. Write $h(w ; s)=r w+\sum_{j=0}^{n} a_{j}(s) w^{-j}$ and suppose that $\gamma\left(s_{0}\right)=\left\{h\left(w ; s_{0}\right),|w|=1\right\}$ is a simple closed polynomial curve of degree $n$ for some $s_{0} \in(0,1]$. Then, for each $s \in(0,1]$, the $\gamma(s)$ remains a simple polynomial curve and $h(w ; s)$, as a map from the exterior of the unit disk into the exterior of $\gamma(s)$, is biholomorphic (a Riemann map). Furthermore, for every $s \in[\delta, 1], 0<\delta<1, t_{0}(s)$ is bounded away from zero.

Proof. Let us begin with the estimation of $t_{0}$. It follows from (25) that $r>\left|a_{j}(s)\right|$ holds for every $j$. Multiplying $\xi(s)$ by $r$ it yields that

$$
0<r^{2}-\sum_{j=1}^{n} j\left|a_{j}(s)\right| r<r^{2}-\sum_{j=1}^{n} j\left|a_{j}(s)\right|^{2}=t_{0}(s)
$$


is bounded away from zero for $s \in[\delta, 1], 0<\delta<1$. Eq. (25) also implies that $h(w ; s)$ is an analytic curve, that is, the derivative of $h(w ; s)$ with respect to $w$, denoted by $h^{\prime}(w ; s)$ is bounded away from zero:

$$
\left|h^{\prime}(w ; s)\right|=\left|r-\sum_{j=1}^{n} j a_{j}(s) w^{-(j+1)}\right| \geq r-\sum_{j=1}^{n} j\left|a_{j}(s)\right||w|^{-(j+1)},
$$

where in the last passage we have used the triangular inequality. Since we are analyzing the exterior of the unity circle $|w|>1$, we have

$$
\left|h^{\prime}(w ; s)\right| \geq r-\sum_{j=1}^{n} j\left|a_{j}(s)\right|>0, \quad \forall s \in(0,1],
$$

which also holds in a small neighborhood of $|w|=1$.

Now, for every $w, z \in \mathbb{C}$ with $|w|=|z|=1$, by the triangular inequality,

$$
\begin{aligned}
|h(w ; s)-h(z ; s)| & \geq r|w-z|-\sum_{j=1}^{n}\left|a_{j}(s)\right|\left|\frac{1}{w^{j}}-\frac{1}{z^{j}}\right| \\
& =r|w-z|-\sum_{j=1}^{n}\left|a_{j}(s)\right|\left|w^{j}-z^{j}\right| \\
& \geq \xi(s)|w-z|>0
\end{aligned}
$$

if $w \neq z$. The last passage follows from $\left|w^{j}-z^{j}\right| \leq j|w-z|$ which can be shown using the telescopic identity

$$
w^{j}-z^{j}=w^{j-1}(w-z)+w^{j-2}(w-z) z+\cdots+(w-z) z^{j-1}
$$

together with the triangular inequality. Equations (27) and (26) imply that the map $h(\cdot ; s): S^{1} \longrightarrow \mathbb{C}$ is an embedding, $\gamma(s)$ is a simple curve and $h(w ; s)$ is a Riemann map from the exterior of the unit circle onto the exterior of $\gamma(s)$ for every $s \in(0,1]$, by continuity. The polynomial curve $\gamma(s)$ with $0<s<1$ preserves all properties assumed for $\gamma\left(s_{0}\right)=\gamma$, concluding the proof of Proposition 1 .

Remark 6 Proposition 1 has an intuitive appeal. To a polynomial curve $\gamma(s)$ fail to be simple it has to develop a cusp. However, when $h(w, s)$ forms a cusp at $w_{c}$, we have $h^{\prime}\left(w_{c}, s\right)=0$, this situation is prevented as long as $\xi(s)>0$. Proposition 1 gives a sufficient condition for $h^{\prime}(w, s) \neq 0$ and show that (25) is also sufficient for $\gamma(s)$ to remain a simple curve.

The next result concerns the conditions $(i-i i i)$ of Remark 3 and the deformation of $\gamma(s)$ to the real line as $s \rightarrow 0$.

Proposition 2 Consider a polynomial curve $\gamma(s)$ of degree $n$ parameterized by $h(w ; s)=r w+\sum_{j=0}^{n} a_{j}(s) w^{-j}$ with $r>0$ and the $\alpha_{j}(s)=r^{-j} a_{j}(s)$ continuous and satisfying conditions $(i-i i i)$ stated in Remark 3. Then $\lim _{s \rightarrow 0} h(w ; s)$ maps the exterior of the unit disk onto $\mathbb{C} \backslash[-2 r, 2 r]$. 
Proof. By the conditions $(i-i i i)$ we have

$$
\begin{aligned}
& a_{0}(s)=\alpha_{0}(s)=o(1), \\
& a_{1}(s)=r+\mathcal{O}(s), \\
& a_{j}(s)=o(s), \quad 2 \leq j \leq n
\end{aligned}
$$

which implies that (25) holds for some $s_{1} \in(0,1]$ small and $\gamma(s)=\{h(w, s),|w|=1\}$ is a simple polynomial curve for every $s \in\left(0, s_{1}\right]$. As $h(w, s)$ is a Riemann map from the exterior of the unit disk into the exterior of $\gamma(s)$ for all $s \in\left(0, s_{1}\right]$ by Proposition 1 , then it suffices to show that $\lim _{s \rightarrow 0} \gamma(s)=[-2 r, 2 r]$. Indeed, since $|w|=1$ we may choose $w=e^{i \theta}$ with $\theta \in[0,2 \pi]$. The Riemann map , under the hypotheses, reads

$$
h\left(e^{i \theta} ; s\right)=r\left(e^{i \theta}+e^{-i \theta}\right)+o(1)
$$

implying that $\lim _{s \rightarrow 0} h\left(e^{i \theta}, s\right)=2 r \cos \theta \in[-2 r, 2 r]$ for all $\theta \in[0,2 \pi]$.

\section{Exterior Harmonic Moments of Near-to-Slit Domains}

Let $\boldsymbol{t}=\left(t_{1}, t_{2}, \ldots, t_{n+1}\right)$ be the exterior harmonic moment of the domain $D$ - containing the origin and bounded by $\gamma-$ and let $\pi t_{0}$ be the area of $D$.

When a given collection $\boldsymbol{t}$ of $n+1$ complex numbers, together with $t_{0}>0$, determines a simple polynomial curve $\gamma$ of degree $n$ ? We refer to Theorem 5.3 of [16] for the solution to this moment problem. If $t_{1}=0$ and $\left|t_{2}\right|<1 / 2$, then every complex number $t_{2}, \ldots, t_{n+1}$ determine a curve $\gamma$ with these properties provided $t_{0}<\delta$, for $\delta$ sufficiently small. We shall give a proof of this result in a language more appropriated for the generalization needed.

The map $(\rho, \boldsymbol{\alpha}) \in \mathbb{R}_{+} \times \mathbb{C}^{n+1} \longmapsto\left(t_{0}, \boldsymbol{t}\right) \in \mathbb{R}_{+} \times \mathbb{C}^{n+1}$ defined by (18) and by the contour integral (5),

$$
\begin{aligned}
j t_{j} & =\frac{1}{2 \pi i} \oint_{|w|=1} \bar{h}\left(w^{-1}\right) h^{\prime}(w) h^{-j}(w) d w \\
& =\sum_{k=j-1}^{n} \bar{\alpha}_{k} \operatorname{Res}\left(w^{k-j} \frac{1-\sum_{l=1}^{n} l \alpha_{l} \rho^{l} / w^{l+1}}{\left(1+\sum_{l=0}^{n} \alpha_{l} \rho^{l} / w^{l+1}\right)^{j}} ; \infty\right)
\end{aligned}
$$

taking residues at infinity:

$$
\begin{aligned}
& t_{0}=\rho-\sum_{j=1}^{n} j\left|\alpha_{j}\right|^{2} \rho^{j} \\
& t_{j}=\frac{1}{j} \bar{\alpha}_{j-1}-\bar{\alpha}_{j} \alpha_{0}-\left(1+\frac{1}{j}\right) \alpha_{1} \bar{\alpha}_{j+1} \rho+O\left(\rho^{2}\right), \quad 1 \leq j \leq n+1
\end{aligned}
$$

with $\rho=r^{2}, \alpha_{j}=r^{-j} a_{j}, 0 \leq j \leq n$, and $\alpha_{j}=0$ if $j>n$, has a smooth inverse in a neighborhood of $\left(0,0, t_{2}, \ldots, t_{n+1}\right)$ provided $\left|t_{2}\right| \neq 1 / 2$.

In this section, we show how the inverse function theorem is applied in this situation and extend it for the case $\left|t_{2}\right|=1 / 2$. We also verify whether the inverse determines a simple polynomial curve $\gamma=\partial D$ and whether a measure $\mu$, uniform in a near-to-slit domain $D$, is the equilibrium measure of the Elbau-Felder ensemble. 


\subsection{Bifurcating Curves}

We observe that $\rho^{*}=\alpha_{0}^{*}=0$ and $\alpha_{j}^{*}=(j+1) \bar{t}_{j+1}, j=1, \ldots, n$ solve the equations $(29)$ for $\left(\rho, \alpha_{0}, \ldots, \alpha_{n}\right)$ and because it takes complex conjugation of the $t_{j+1}$, we look (29) as a map from $\mathbb{R}_{+} \times \mathbb{C}^{n+1} \times \mathbb{C}^{n+1}$ into itself

$$
F:(\rho, \boldsymbol{\alpha}, \overline{\boldsymbol{\alpha}}) \longmapsto\left(t_{0}, \boldsymbol{t}, \overline{\boldsymbol{t}}\right)
$$

and we write

$$
\begin{aligned}
0 & =\frac{F\left(\rho, \boldsymbol{\alpha}^{*}+\rho \boldsymbol{\varphi}, \overline{\boldsymbol{\alpha}}^{*}+\rho \overline{\boldsymbol{\varphi}}\right)-\left(t_{0}, \boldsymbol{t}, \overline{\boldsymbol{t}}\right)}{\rho} \\
& =l\left(\rho^{*}, \boldsymbol{\varphi}, \overline{\boldsymbol{\varphi}}\right)+p(\rho, \boldsymbol{\varphi}, \overline{\boldsymbol{\varphi}})-\left(\tau_{0}, \mathbf{0}, \mathbf{0}\right) .
\end{aligned}
$$

Here, $F$ has been expanded in Taylor series about $\left(\rho^{*}, \boldsymbol{\alpha}^{*}, \overline{\boldsymbol{\alpha}}^{*}\right)$ with remainder $\rho p(\rho, \boldsymbol{\varphi}, \overline{\boldsymbol{\varphi}})=O\left(\rho^{2}\right), \tau_{0}=t_{0} / \rho=O(1)$ and $l$ is the linear map

$$
l\left(\rho^{*}, \boldsymbol{\varphi}, \overline{\boldsymbol{\varphi}}\right)=L\left[\rho^{*}, \boldsymbol{\alpha}^{*}, \overline{\boldsymbol{\alpha}}^{*}\right]\left(\begin{array}{c}
1 \\
\boldsymbol{\varphi} \\
\overline{\boldsymbol{\varphi}}
\end{array}\right)=\left(\begin{array}{ccc}
1-4\left|t_{2}\right|^{2} & \mathbf{0}^{T} & \mathbf{0}^{T} \\
-\overline{\boldsymbol{v}} & -\bar{K} & J^{-1} \\
-\boldsymbol{v} & J^{-1} & -K
\end{array}\right)\left(\begin{array}{c}
1 \\
\boldsymbol{\varphi} \\
\overline{\boldsymbol{\varphi}}
\end{array}\right)
$$

where $\mathbf{0}$ is the null column vector in $\mathbb{C}^{n+1}, \bar{v}_{j}=2(1+1 / j)(j+2) \bar{t}_{2} t_{j+2}$ if $1 \leq j<n$ with $v_{n}=v_{n+1}=0, J=\operatorname{diag}\{j\}_{j=1}^{n+1}$ and $K$ is a $(n+1) \times(n+1)$ matrix with $\bar{K}_{i 1}=(i+1) t_{i+1}$ for $1 \leq i \leq n$ and 0 otherwise.

Since $L$ is invertible for $\left|t_{2}\right| \neq 1 / 2$, (30) has a smooth inverse defined in a neighborhood of $\left(t_{0}, \boldsymbol{t}\right)=\left(0,0, t_{2}, \ldots, t_{n+1}\right)$. The implicit function theorem applied to (31) (with $\boldsymbol{t} \in \mathbb{C}^{n+1}$ fixed) uniquely defines two smooth curves parameterized by $\rho$ :

$$
\begin{aligned}
& \boldsymbol{\varphi}(\rho)=T \boldsymbol{v}+B \overline{\boldsymbol{v}}+\mathcal{O}(\rho) \\
& \tau_{0}(\rho)=1-4\left|t_{2}\right|^{2}+\mathcal{O}(\rho)
\end{aligned}
$$

on $\mathbb{C}^{n+1}$ and $\mathbb{R}_{+}$, respectively, where $B=\left(1-4\left|t_{2}\right|^{2}\right)^{-1} J K$ and $T=J+2 \bar{t}_{2} B$. We note that the leading order in $\rho$ of equation (31) can be written as

$$
\begin{aligned}
& 1-4\left|t_{2}\right|^{2}=\tau_{0} \\
&\left(\begin{array}{cc}
-\bar{K} & J^{-1} \\
J^{-1} & -K
\end{array}\right)\left(\begin{array}{l}
\boldsymbol{\varphi} \\
\overline{\boldsymbol{\varphi}}
\end{array}\right)=\left(\begin{array}{l}
\overline{\boldsymbol{v}} \\
\boldsymbol{v}
\end{array}\right)
\end{aligned}
$$

whose solution is given in Appendix A.

The function $t_{0}=\rho \tau_{0}(\rho)$ is monotone increasing in $\rho \in[0, a)$ for $\left|t_{2}\right|<1 / 2$ and sufficiently small $a$, and its inverse, $\rho\left(t_{0}, \boldsymbol{t}\right)$, is a well defined function of $t_{0}$ and $\boldsymbol{t}$. The inverse of $(29)$ in $\mathbb{R}_{+} \times \mathbb{C}^{n+1}$ thus reads

$$
\left(t_{0}, \boldsymbol{t}\right) \longmapsto\left(\rho\left(t_{0}, \boldsymbol{t}\right), \boldsymbol{\alpha}^{*}+\rho\left(t_{0}, \boldsymbol{t}\right) \boldsymbol{\varphi} \circ \rho\left(t_{0}, \boldsymbol{t}\right)\right) .
$$

The above application of the implicit function theorem breaks down if the second harmonic moment $t_{2}$ tends to $1 / 2$ and this is the case when the external domain of $\gamma(s)$ tends, as $s \rightarrow 0$, to the slit domain $\mathbb{C} /[-2 r, 2 r]$ (see Proposition 2). We need, 
therefore, to extend Theorem 5.3 of [16] to include this case. For this, we shall rescale all components of $\boldsymbol{t}$, excepted $t_{2}$ whose modulus square will be denoted by $\lambda=\left|t_{2}\right|^{2}$, as $\ddagger$

$$
\begin{aligned}
& t_{2}=\sqrt{\lambda} \tau_{2}=\sqrt{\lambda} e^{i \arg \tau_{2}} \\
& t_{j}=(1-4 \lambda) \tau_{j}, 2<j \leq n+1
\end{aligned}
$$

and apply the constructive bifurcation theory from a simple eigenvalue developed by Crandall and Rabinowitz (see e.g. [17]). The theory applied to equation (31) uses the implicit function theorem with the role of $\rho$ and $\lambda$ exchanged. Instead of the two parametric curves $\varphi_{j}=\varphi_{j}(\rho)$ and $\tau_{0}=\tau_{0}(\rho)$, we consider $\tilde{\varphi}_{j}=\tilde{\varphi}_{j}(\rho, \lambda)$ and $\tilde{\tau}_{0}=\tilde{\tau}_{0}(\rho, \lambda)$ as a function of $\lambda$ for $(\rho, \boldsymbol{t}) \in \mathbb{C}^{n+1}$ fixed, where $\boldsymbol{\tau}=\left(\tau_{1}, \tau_{2}, \ldots, \tau_{n+1}\right)$ is a vector in the affine space of $\mathbb{C}^{n+1}$ with $\left|\tau_{2}\right|=1$, denoted by $Z$.

We observe that $\left(\rho^{*}, \boldsymbol{\alpha}^{*}\right)$ with $\left|t_{2}\right|=1 / 2$ is a bifurcation point for (29) since every neighborhood of this point contains a solution which differs from (34). Note that the tangent map of $(29)$ at $\left(\rho^{*}, \boldsymbol{\alpha}^{*}\right)$ with $\left|t_{2}\right|=1 / 2$ is singular, i. e., $L$ is not invertible at $\lambda=\left|t_{2}\right|^{2}=1 / 4$. Using bifurcation theory, we shall construct a pair of smooth curves for fixed $(\rho, \boldsymbol{\tau}) \in \mathbb{C}^{n+1}: \tilde{\varphi}_{j}=\tilde{\varphi}_{j}(\lambda)$ and $\tilde{\tau}_{0}=\tilde{\tau}_{0}(\lambda), \lambda \in(1 / 4-b, 1 / 4]$ for some $b>0$, such that $\tilde{\varphi}_{j}(1 / 4)=\varphi_{j}\left(\rho=0,\left|t_{2}\right|=1 / 2\right)$ and $\tilde{\tau}_{0}(1 / 4)=\tau_{0}\left(\rho=0,\left|t_{2}\right|=1 / 2\right)$.

Proposition 3 Given $(\rho, \boldsymbol{\tau}) \in \mathbb{R}_{+} \times Z \simeq \mathbb{C}^{n+1}$, there exist two uniquely defined smooth curves, $\lambda \mapsto \boldsymbol{\zeta}(\rho, \lambda, \boldsymbol{\tau})$ on $\mathbb{C}^{n+1}$ and $\lambda \mapsto \tilde{\tau}_{0}(\rho, \lambda, \boldsymbol{\tau})$ on $\mathbb{R}$, defined by (41), such that the solution of (29) for $(\rho, \boldsymbol{\alpha})$ in a neighborhood of $\left(t_{0}, \rho, \boldsymbol{\tau}\right)=(0,0, \boldsymbol{\tau})$ in $\mathbb{R}_{+} \times \mathbb{R}_{+} \times Z$ can be written as

$$
\left(t_{0}, \rho, \boldsymbol{\tau}\right) \longmapsto\left(\rho, \boldsymbol{\alpha}^{*} \circ \lambda\left(t_{0}, \rho, \boldsymbol{\tau}\right)+\rho\left(c \boldsymbol{\alpha}_{0}+\boldsymbol{\zeta}\right) \circ \lambda\left(t_{0}, \rho, \boldsymbol{\tau}\right)\right)
$$

where $\lambda\left(t_{0}, \rho, \boldsymbol{\tau}\right)$ is a function of $t_{0}, \rho$ and $\boldsymbol{\tau}$ which is the unique solution for $\lambda$ of $t_{0}=\rho \tilde{\tau}_{0}(\rho, \lambda, \boldsymbol{\tau})$ in the domain $0<\lambda \leq 1 / 4, \rho \in\left[0, \hat{r}^{2}\right]$ with $\hat{r}=\hat{r}(\boldsymbol{\tau})$ sufficiently small and $\left(c \boldsymbol{\alpha}_{0}\right)_{1}=12\left(\bar{\tau}_{2} \tau_{3}+\bar{\tau}_{3}\right) /\left(\tau_{2}+\bar{\tau}_{2}\right)$ and $\left(c \boldsymbol{\alpha}_{0}\right)_{j}=0$ otherwise. Moreover, there exists $\bar{r}=\bar{r}(\boldsymbol{\tau})>0$ such that $h(w)$, defined by (9) with $a_{j}=r^{j} \alpha_{j}$ and $r^{2}=\rho$ where $(\rho, \boldsymbol{\alpha})$ is given by (36), parameterizes a simple polynomial curve of order $n$ which can be deformed into the segment $\left[-2 r+\alpha_{0}, 2 r+\alpha_{0}\right]$, for every fixed $r<\bar{r}$, provided $\tau_{2}=1$.

Proof. It suffices, for the first statement, to verify the hypothesis of Theorem 1 in Sec. 3 of [17]. For this, it is convenient to include in the linear map (32) higher order in $\rho$ terms. So, let

$$
\tilde{l}(\rho, \boldsymbol{\varphi}, \overline{\boldsymbol{\varphi}})=\lim _{\varepsilon \rightarrow 0} \frac{1}{\varepsilon}\left(F\left(\rho+\varepsilon, \boldsymbol{\alpha}^{*}+\varepsilon \boldsymbol{\varphi}, \overline{\boldsymbol{\alpha}}^{*}+\varepsilon \overline{\boldsymbol{\varphi}}\right)-F\left(\rho, \boldsymbol{\alpha}^{*}, \overline{\boldsymbol{\alpha}}^{*}\right)\right)
$$

and write (31) as

$$
l_{0}(\rho, \boldsymbol{\varphi}, \overline{\boldsymbol{\varphi}})+(1-4 \lambda) l_{1}(\rho, \boldsymbol{\varphi}, \overline{\boldsymbol{\varphi}})+\tilde{p}(\rho, \lambda, \boldsymbol{\varphi}, \overline{\boldsymbol{\varphi}})-\left(\tilde{\tau}_{0}, \mathbf{0}, \mathbf{0}\right)
$$

$\ddagger$ We use concomitantly the notation $s=1-4 \lambda$ adopted in the introduction (see eqs. (10)). 
where $\tilde{\tau}_{0}=t_{0} / \rho, l_{0}$ and $l_{1}$ are linear maps in $\mathbb{R}_{+} \times \mathbb{C}^{n+1} \times \mathbb{C}^{n+1}$, with $l_{0}=\left.\tilde{l}\right|_{\lambda=1 / 4}$ and $4 l_{1}=-\partial \tilde{l} /\left.\partial \lambda\right|_{\lambda=1 / 4}$, hence:

$$
\begin{aligned}
& l_{0}(\rho, \boldsymbol{\varphi}, \overline{\boldsymbol{\varphi}})=L_{0}\left(\begin{array}{c}
1 \\
\boldsymbol{\varphi} \\
\overline{\boldsymbol{\varphi}}
\end{array}\right)=\left(\begin{array}{ccc}
0 & \overline{\boldsymbol{v}}_{0}^{T} & \boldsymbol{v}_{0}^{T} \\
\mathbf{0} & -\bar{K}_{0} & J^{-1}+\bar{M}_{0} \\
\mathbf{0} & J^{-1}+M_{0} & -K_{0}
\end{array}\right)\left(\begin{array}{c}
1 \\
\boldsymbol{\varphi} \\
\overline{\boldsymbol{\varphi}}
\end{array}\right) \\
& l_{1}(\rho, \boldsymbol{\varphi}, \overline{\boldsymbol{\varphi}})=L_{1}\left(\begin{array}{c}
1 \\
\boldsymbol{\varphi} \\
\overline{\boldsymbol{\varphi}}
\end{array}\right)=\left(\begin{array}{ccc}
1 & \mathbf{0}^{T} & \mathbf{0}^{T} \\
-\overline{\boldsymbol{v}}_{1} & -\bar{K}_{1} & O \\
-\boldsymbol{v}_{1} & O & -K_{1}
\end{array}\right)\left(\begin{array}{c}
1 \\
\boldsymbol{\varphi} \\
\overline{\boldsymbol{\varphi}}
\end{array}\right)
\end{aligned}
$$

with $\overline{\boldsymbol{v}}_{0}=\mathcal{O}(\rho),\left(\overline{\boldsymbol{v}}_{1}\right)_{j}=(1+1 / j)(j+2) \tau_{j+2} / \tau_{2}+\mathcal{O}(\rho),\left(\bar{K}_{0}\right)_{i j}=\tau_{2} \delta_{i 1} \delta_{j 1}, M_{0}=\mathcal{O}(\rho)$ and, given

$$
\overline{\boldsymbol{w}}_{1}=\left(-\tau_{2} / 2,3 \tau_{3}, \ldots,(n+1) \tau_{n+1}, 0\right)
$$

$\bar{K}_{1}$ is, up to the leading order in $\rho$, a $(n+1) \times(n+1)$ matrix with $\overline{\boldsymbol{w}}_{1}$ in its first column and 0 everywhere else; $O$ denotes the $(n+1) \times(n+1)$ null matrix. Moreover, $\tilde{p}(\rho, \lambda, \boldsymbol{\varphi}, \overline{\boldsymbol{\varphi}})$ is a smooth map from $\mathbb{R}_{+} \times \mathbb{R}_{+} \times \mathbb{C}^{n+1} \times \mathbb{C}^{n+1}$ to $\mathbb{R}_{+} \times \mathbb{C}^{n+1} \times \mathbb{C}^{n+1}$ which satisfies

$$
\tilde{p}(\rho, 1 / 4, \mathbf{0}, \mathbf{0})=\frac{\partial \tilde{p}}{\partial \lambda}(\rho, 1 / 4, \mathbf{0}, \mathbf{0})=0
$$

and

$$
\frac{\partial^{2} \tilde{p}}{\partial \boldsymbol{\varphi} \partial \lambda}(\rho, 1 / 4, \mathbf{0}, \mathbf{0})=\frac{\partial^{2} \tilde{p}}{\partial \overline{\boldsymbol{\varphi}} \partial \lambda}(\rho, 1 / 4, \mathbf{0}, \mathbf{0})=0 .
$$

We observe that (31), and consequently (37), extends by continuity to $\rho=0$. In this way, we define

$$
G(\rho, \lambda, \boldsymbol{\zeta}, \overline{\boldsymbol{\zeta}})=\frac{F\left(\rho, \boldsymbol{\alpha}^{*}+\rho\left(c \boldsymbol{\alpha}_{0}+\boldsymbol{\zeta}\right), \overline{\boldsymbol{\alpha}}^{*}+\rho\left(c \overline{\boldsymbol{\alpha}}_{0}+\overline{\boldsymbol{\zeta}}\right)\right)-\left(t_{0}, \boldsymbol{t}, \overline{\boldsymbol{t}}\right)}{\rho}
$$

for $(\rho, \lambda, \boldsymbol{\zeta}, \overline{\boldsymbol{\zeta}}) \in[0, a) \times(1 / 4-b, 1 / 4] \times \mathbb{C}^{n+1} \times \mathbb{C}^{n+1}$, where $\boldsymbol{\alpha}_{0}=\left(1 / \sqrt{2 \tau_{2}}, 0, \ldots, 0\right)$ and $c$ is a constant to be properly chosen as we shall discuss. We observe that $\boldsymbol{e}_{0}=\left(\boldsymbol{\alpha}_{0}, \overline{\boldsymbol{\alpha}}_{0}\right)$ is an eigenvector of

$$
A_{0}=\left(\begin{array}{cc}
-\bar{K}_{0} & J^{-1} \\
J^{-1} & -K_{0}
\end{array}\right)
$$

the matrix $L_{0}$ with the first line and column removed and the $\mathcal{O}(\rho)$ corrections omitted, associated with the (simple) null eigenvalue. It can be shown by (29) and (28) that the $\mathcal{O}(\rho)$ corrections do not affect the entry $\left(K_{0}\right)_{11}=\bar{\tau}_{2}$ and $\left(J^{-1}\right)_{11}=1 . \S$ So, the null eigenvalue and its eigenvector $\boldsymbol{e}_{0}$ remain unaltered for $\rho>0$.

It follows by (37)-(39), that

$$
G(\rho, \lambda, \boldsymbol{\zeta}, \overline{\boldsymbol{\zeta}})=l_{0}(0, \boldsymbol{\zeta}, \overline{\boldsymbol{\zeta}})+(1-4 \lambda) l_{1}\left(1, c \boldsymbol{\alpha}_{0}+\boldsymbol{\zeta}, c \overline{\boldsymbol{\alpha}}_{0}+\overline{\boldsymbol{\zeta}}\right)-\left(\tau_{0}, \mathbf{0}, \mathbf{0}\right)+\hat{p}(\lambda, \rho, \boldsymbol{\zeta}, \overline{\boldsymbol{\zeta}})
$$

$\S$ The nonvanishing elements of $\boldsymbol{v}_{0}$ and $M_{0}$ in (38) are, respectively, $\left(\boldsymbol{v}_{0}\right)_{2}=\tau_{2} \rho$ and $M_{i, i+2}=$ $-\tau_{2}(1+1 / i) \rho / 2$ for $1 \leq i \leq n-1$. 
with $\hat{p}(\lambda, \rho, \boldsymbol{\zeta}, \overline{\boldsymbol{\zeta}})=\tilde{p}\left(\lambda, \rho, c \boldsymbol{\alpha}_{0}+\boldsymbol{\zeta}, c \overline{\boldsymbol{\alpha}}_{0}+\overline{\boldsymbol{\zeta}}\right)$, and the implicit function theory can be applied to $G=0$ provided the derivative of $G(\rho, \lambda, \boldsymbol{\zeta}, \overline{\boldsymbol{\zeta}})$ about $(\lambda, \boldsymbol{\zeta}, \overline{\boldsymbol{\zeta}})=(1 / 4, \mathbf{0}, \mathbf{0})$ defined by the linear map

$$
(\lambda, \boldsymbol{\zeta}, \overline{\boldsymbol{\zeta}}) \mapsto l_{0}(\rho, \boldsymbol{\zeta}, \overline{\boldsymbol{\zeta}})-4 \lambda l_{1}\left(\rho, \boldsymbol{\alpha}_{0}, \overline{\boldsymbol{\alpha}}_{0}\right)=\left(\begin{array}{ccc}
-4 & \overline{\boldsymbol{v}}_{0}^{T} & \boldsymbol{v}_{0}^{T} \\
4\left(\overline{\boldsymbol{v}}_{1}+c \overline{\boldsymbol{w}}_{1} / \sqrt{2 \tau_{2}}\right) & -\bar{K}_{0} & J^{-1}+\bar{M}_{0} \\
4\left(\boldsymbol{v}_{1}+c \boldsymbol{w}_{1} \sqrt{\tau_{2} / 2}\right) & J^{-1}+M_{0} & -K_{0}
\end{array}\right)\left(\begin{array}{c}
\lambda \\
\boldsymbol{\zeta} \\
\overline{\boldsymbol{\zeta}}
\end{array}\right)
$$

is nonsingular (see Theorem 1 in Sec. 3 of [17]). Since $A_{0}$ is singular, the linear map is invertible if and only if $A_{0}^{-1}$ applies to vectors in $\mathbb{C}^{n+1} \times \mathbb{C}^{n+1}$ orthogonal to $\boldsymbol{e}_{0}$. For this, we pick $c$ so that $\overline{\boldsymbol{v}}_{1}+c \overline{\boldsymbol{w}}_{1} / \sqrt{2 \tau_{2}}$ is orthogonal (with respect to the inner product $\left.\boldsymbol{u} \cdot \boldsymbol{v}=\sum_{i=1}^{n+1}\left(\bar{u}_{i} v_{i}+u_{i} \bar{v}_{i}\right)\right)$ to $\boldsymbol{\alpha}_{0}:$

$$
c=12 \sqrt{2} \frac{\sqrt{\overline{\tau_{2}}} \tau_{3}+\sqrt{\tau_{2}} \bar{\tau}_{3}}{\tau_{2}+\bar{\tau}_{2}} .
$$

With this choice, $G=0$ (with $\rho>0$ and $\tau \in Z$ fixed) uniquely defines two smooth parametric curves:

$$
\begin{aligned}
& \boldsymbol{\zeta}(\lambda)=(1-4 \lambda)\left(J\left(\boldsymbol{v}_{1}+c \boldsymbol{w}_{1} \sqrt{\tau_{2} / 2}\right)+\mathcal{O}(\rho)\right)+\mathcal{O}\left((1-4 \lambda)^{2}\right) \\
& \tilde{\tau}_{0}(\lambda)=1-4 \lambda+\mathcal{O}\left((1-4 \lambda)^{2}\right)
\end{aligned}
$$

on $\mathbb{C}^{n+1}$ and on $\mathbb{R}_{+}$. As in the previous case, the leading order in $s=1-4 \lambda$ of equation $G=0$ reads

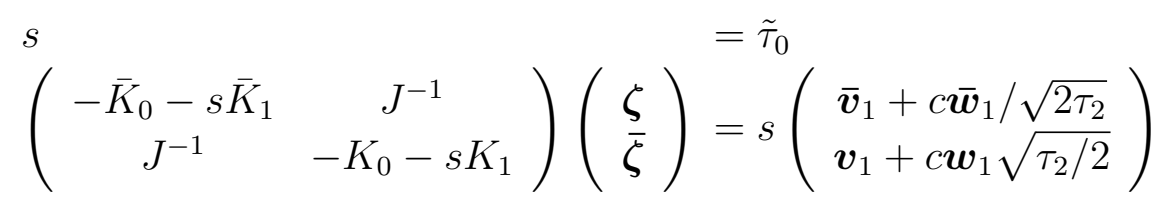

from where (41) can be readily obtained using Lemma 2.

The function $t_{0}=\rho \tilde{\tau}_{0}(\rho, \lambda)$ is monotone decreasing in $\lambda$, for $1 / 4-b<\lambda \leq 1 / 4$, $\rho \in\left(0, \hat{r}^{2}\right]$ with $\hat{r}$ sufficiently small, and its inverse, $\lambda\left(t_{0}, \rho, \boldsymbol{\tau}\right)$, is a well defined function of $t_{0}, \rho$ and $\boldsymbol{\tau}$. The solution of $(29)$ for $(\rho, \boldsymbol{\alpha})$ in a neighborhood of $\left(t_{0}, \rho, \boldsymbol{\tau}\right)=(0,0, \boldsymbol{\tau})$ in $\mathbb{R}_{+} \times \mathbb{R}_{+} \times Z$ is thus given by (36). From the first equation of (29), together with (45) bellow and (41), if $\hat{r}=\hat{r}(\boldsymbol{\tau})$ is sufficiently small,

$$
\begin{aligned}
t_{0} & =s \rho\left(1-s \sum_{j=2}^{n} j(j+1)^{2}\left|\tau_{j+1}\right|^{2} \rho^{j-1}+\mathcal{O}\left(s^{2}, s \rho^{2}\right)\right) \\
& =s \rho\left(1-18 s \rho+\mathcal{O}\left(s^{2}, s \rho^{2}\right)\right)
\end{aligned}
$$

is monotone in $s$ provided $s<\frac{1}{36 \hat{r}^{2}}<\frac{1}{36 \rho}$ so, the dependence of $b$ on $\rho$ is estimated by $b>\frac{1}{144 \hat{r}^{2}}$. We choose $\hat{r}$ so that $t_{0}$ becomes a monotone function of $\lambda$ in the whole domain $(0,1 / 4]$.

To conclude the proof, it remains to verify that

$$
h(w)=r w+\alpha_{0}+r \frac{\alpha_{1}}{w}+\cdots+r^{n} \frac{\alpha_{n}}{w^{n}},|w|=1
$$


with

$$
\alpha_{j}=\alpha_{j}^{*} \circ \lambda\left(t_{0}, \rho, \boldsymbol{\tau}\right)+\rho\left(c\left(\boldsymbol{\alpha}_{0}\right)_{j+1}+\zeta_{j+1} \circ \lambda\left(t_{0}, \rho, \boldsymbol{\tau}\right)\right)
$$

parameterizes a simple polynomial curve $\gamma=\gamma\left(t_{0}, \rho, \boldsymbol{\tau}\right)$. For this, in order $\gamma$ to approach a segment $I$, as the area of its interior $\pi t_{0}$ tends to 0 , we let $\tau_{2}, \tau_{3}$ and $\rho=r^{2}$ so that

$$
\alpha_{0}=\rho\left(c\left(\boldsymbol{\alpha}_{0}\right)_{1}+\zeta_{1}\right)=12 \frac{\bar{\tau}_{2} \tau_{3}+\bar{\tau}_{3}}{\tau_{2}+\bar{\tau}_{2}} r^{2}+\mathcal{O}\left(t_{0} / r^{2}\right)
$$

remains closed to the origin.\| Now, it is enough to verify the hypothesis (25) of Proposition 1. It follows immediately from (45) and (43) that

$$
\begin{aligned}
& \alpha_{1}=1-\frac{t_{0}}{2 r^{2}}+\mathcal{O}\left(t_{0}\right) \\
& \alpha_{j}=\frac{t_{0}}{r^{2}}(j+1) \bar{\tau}_{j+1}+\mathcal{O}\left(t_{0}\right), \quad 2 \leq j \leq n
\end{aligned}
$$

for every $\tau$, and

$$
\xi=r-\sum_{j=1}^{n} j r^{j}\left|\alpha_{j}\right|=\frac{t_{0}}{r^{2}}\left(\frac{r}{2}-\sum_{j=2}^{n} j(j+1) r^{j}\left|\tau_{j+1}\right|\right)+\mathcal{O}\left(t_{0}\right)>0
$$

holds for $r<\bar{r}$ where $\bar{r}=\bar{r}(\boldsymbol{\tau})$ is the largest value of $r$ that still makes the above expression positive, for $t_{0}$ small enough.

Remark $7 r_{0}$ in Theorem 1 is thus given by $\min (\bar{r}, \hat{r})$.

Corollary 1 If (35) is scaled with a different power of $s=(1-4 \lambda): t_{j}=s^{\Delta_{j}} \tau_{j}, \Delta_{j}>1$ for each $2<j \leq n+1$, then (45) behaves as $\alpha_{0}(s)=o(1), \alpha_{1}(s)=1-s / 2+o(s)$ and $\alpha_{j}(s)=o(s), 2 \leq j \leq n$, as $s$ tends to 0 .

Proof: Proposition 3 can be adapted for (35) scaled with different power of $s=(1-4 \lambda)$ as follows. Replacing the $\tau_{j}$ in (41) by a $o(1)$ function of $s$, every component $\zeta_{j}$ with $j \neq 1$ becomes $o(s)$. $\boldsymbol{\alpha}^{*}$ becomes $o(s)$, likewise. By continuity, the claimed behavior of $\alpha_{j}$, with $1 \leq j \leq n$, can be read directly from (45). $\alpha_{0}$ is $o(1)$ by (46).

Note that Proposition 3 holds for simple closed analytic curves provided $l, l_{0}$ and $l_{1}$ are Frechét derivatives of $F$ with respect to an appropriate Banach space.

\subsection{Equilibrium Measure}

Equation (21) is equivalent to

$$
0 \leq \mathcal{E}(w):=E(h(w))=\frac{1}{t_{0}}\left(|h(w)|^{2}-|h(1)|^{2}+2 \Re \mathrm{e} \int_{1}^{w} \bar{h}\left(\zeta^{-1}\right) h^{\prime}(\zeta) d \zeta\right)
$$

|| For the parameterization (35), i. e., equation (10) with $s=1-4 \lambda$ and the $\Delta_{j}=1$, $\alpha_{0}$ does not necessarily go to 0 as the curve $\gamma$ collapses to a segment of a line and $\alpha_{0}$ is real only for $\tau_{2}=1$. Note that, by (44), $\alpha_{0}=0$ makes a symmetric (with respect to both, the real and imaginary axis) curve centered at the origin. If we accelerate the rate under which $t_{j}$ in (35) goes to 0 (taking the $\Delta_{j}>1$ in (10)), $\gamma$ recovers its symmetry when it collapses to a segment of the real line. 
for $w \in h^{-1}(\Sigma)$ such that $|w| \geq 1$ (see eq. (16) of [16]), and it suffices to verify only for $|w| \geq 1 / R$, where $R=\max \left\{|w|: h^{\prime}(w)=0, w \in \mathbb{C}\right\}$ is the critical radius of $\gamma$, and $r$ sufficiently small.

Let $h^{(0)}(w)=r w+\alpha_{0}+r \alpha_{1} / w$ be the parameterization of an ellipse that approximates $\gamma$ and let $\left(t_{0}^{(0)}, t_{2}^{(0)}\right)$ denote the corresponding external harmonic moments. We denote by $\mathcal{E}^{(0)}(w)$ the function defined in (49) for the ellipse and note that, by Subsection 6.2 of [16], $t_{0}^{(0)} \mathcal{E}^{(0)}(w)$ remains bounded for $w \in h^{-1}(\Sigma)$ such that $|w| \geq 1$. For $\left(1-t_{0} / r^{2}\right)^{-1} \leq|w|<r^{-\alpha}, 0<\alpha<1 / 3$, we have

$$
\begin{aligned}
\frac{1}{t_{0}}\left(h\left(w^{-1}\right)-h^{(0)}\left(w^{-1}\right)\right) h^{\prime}(w) & =\frac{1}{t_{0}} \frac{r^{2} \alpha_{2}}{w^{-2}} r+\mathcal{O}\left(t_{0}\right)=\mathcal{O}\left(r^{1-2 \alpha}\right) \\
\frac{1}{t_{0}} h^{(0)}\left(w^{-1}\right)\left(h^{\prime}(w)-h^{(0) \prime}(w)\right) & =\frac{1}{t_{0}} r \alpha_{1} w \frac{-2 r^{2} \alpha_{2}}{w^{3}}+\mathcal{O}\left(t_{0}\right)=\mathcal{O}(r) \\
\frac{t_{0}-t_{0}^{(0)}}{t_{0}^{2}} & =\frac{1}{t_{0}^{2}} r^{4} \alpha_{2}^{2}+\mathcal{O}\left(t_{0}\right)=\mathcal{O}(1) \\
t_{0} \mathcal{E}^{(0)}(w) & =\mathcal{O}\left(r^{2-\alpha}\right)
\end{aligned}
$$

uniformly in $t_{0}$ as $t_{0} \rightarrow 0$. Consequently,

$$
\begin{aligned}
|\mathcal{E}(w)| \geq & \frac{1}{t_{0}}\left|t_{0} \mathcal{E}^{(0)}(w)\right|-\left|\mathcal{E}(w)-\mathcal{E}^{(0)}(w)\right| \\
\geq & \frac{1}{t_{0}}\left|t_{0} \mathcal{E}^{(0)}(w)\right|-\left.\frac{1}{t_{0}}|| h(w)\right|^{2}-\left|h^{(0)}(w)\right|^{2}-|h(1)|^{2}+\left|h^{(0)}(1)\right|^{2} \mid \\
& -\frac{2}{t_{0}}\left|\Re \mathrm{e} \int_{1}^{w}\left(h\left(\zeta^{-1}\right)-h^{(0)}\left(\zeta^{-1}\right)\right) h^{\prime}(\zeta) d \zeta\right| \\
& -\frac{2}{t_{0}}\left|\Re \mathrm{e} \int_{1}^{w} h\left(\zeta^{-1}\right)\left(h^{\prime}(\zeta)-h^{(0) \prime}(\zeta)\right) d \zeta\right|-\left|\frac{t_{0}-t_{0}^{(0)}}{t_{0}^{2}} t_{0} \mathcal{E}^{(0)}(w)\right|
\end{aligned}
$$

is strictly positive for $t_{0}$ sufficiently small. For $w \in h^{-1}(\Sigma)$ such that $|w| \geq r^{-\alpha}$ we may proceed exactly as in [16].

\section{Explicit Balayage Measures}

To explicitly obtain the balayage measure in terms of the potentials we need an auxiliary result.

Theorem 3 If $V: \Sigma \rightarrow \mathbb{R}$ is a potential defined in a compact set $\Sigma \subset \mathbb{C}$ with continuous second partial derivatives in its interior, then the variational problem (15) is attained at a unique equilibrium measure $\mu^{V}$ supported in a compact set $D \subset \Sigma$ given by

$$
d \mu^{V}(z)=\frac{1}{4 \pi} \Delta V d^{2} z
$$

at almost every (with respect to the Lebesgue measure $d^{2} z$ ) interior point of $D$, where $\Delta=\partial^{2} / \partial x^{2}+\partial^{2} / \partial y^{2}$ is the Laplace operator.

By using the smoothness of $V$ the result follows from Theorems $I-1.3$ pp. 27 and $I I-1.3$ pp. 85 of [19]. We derive an explicit equation for the balayage measure in 
terms of the potential $V$. The result will be of fundamental importance to establish the conformal deformation of the Elbau-Felder ensemble.

A direct application of this theorem to the potential $V$ shows that the density of eigenvalues is indeed uniform within $D$. The Elbau-Felder potential reads $V(z)=(z \bar{z}-$ $p(z)-p \overline{(z})) / t_{0}$, remembering that $\Delta=4 \partial_{z} \partial_{\bar{z}}$ and $p(z)$ is analytic $\left(\partial_{\bar{z}} p(z)=\partial_{z} \overline{p(z)}=0\right)$, it follows that $d \mu(z)=1 /\left(\pi t_{0}\right) d^{2} z$ within $D$. Moreover, we have the following result

Theorem 4 Let $V$ be an Elbau-Felder potential. The balayage measure $\widehat{\mu}$ associated with the equilibrium measure $\mu^{V}$ with support on $\gamma=\partial D$ is

$$
d \widehat{\mu}(z)=\frac{1}{2 \pi i t_{0}} S(z) d z
$$

where $d z$ is the measure of arc length on $\gamma$.

Proof: The main ingredient is the Green theorem. Our hypotheses on $V, D$ and $\partial D$ guarantee that the Green theorem is applicable. Using that $\Delta=4 \partial_{z \bar{z}}$ and $f \in \mathcal{H}(D)$, by (50) and applying the Green theorem we have

$$
\begin{aligned}
\int_{D} f(z) d \mu^{V}(z) & =\frac{1}{\pi} \int_{D} \partial_{\bar{z}}\left(f(z) \partial_{z} V(z)\right) d^{2} z \\
& =\frac{1}{2 i \pi} \int_{\partial D} f(z) \partial_{z} V(z) d z
\end{aligned}
$$

and by the balayage measure property Eq. (23), we have

$$
\int_{\partial D} f(z) d \widehat{\mu}^{V}(z)=\frac{1}{2 i \pi} \int_{\partial D} f(z) \partial_{z} V(z) d z
$$

holds for every continuous function on $\partial D$, from where it follows the continuity of the balayage measure with respect to the Lebesgue measure. Next, we have that

$$
d \hat{\mu}(z)=\frac{1}{2 \pi i} \partial_{z} V(z) d z=\frac{1}{t_{0}}\left(\bar{z}-\sum_{k=2}^{n+1} k t_{k} z^{k-1}\right) d z
$$

the term of the sum does not contribute to a contour integral with respect to $\widehat{\mu}$ for test functions $f \in \mathcal{H}(D)$ (by continuity and by the Cauchy theorem). Then, by using the definition $\bar{z}=S(z), z \in \gamma$, of the Schwarz function we obtain Eq. (51), concluding the proof.

Eq. (51) relates the equilibrium measure with the Schwarz function of boundary of the support. We shall conclude, by applying the Cauchy theorem, that only the branch cut in the interior domain $D$ of the Schwarz function will contribute to the balayage measure. Thus, questions concerning the equilibrium measure $\mu$ turns to the behavior of the Schwarz function. It turns out that, unless $\gamma$ is a line or a circle arc, the Schwarz function $S$ always has a branch cut [20]. Our next result draws some conclusions on the behavior of the branch cuts.

Proposition 4 If $\gamma$ is a simple closed analytic curve, then the Schwarz function $S$ associated with $\gamma$ must have branching points in its interior. 
Proof. Take $f \in \mathcal{H}(D)$ such that $\int_{D} f(z) d \mu \neq 0$. By the property (23) of balayage measure with the Theorem 4, we have

$$
\int_{D} f(z) d \mu(z)=\frac{1}{2 \pi i t_{0}} \oint_{\gamma} f(z) S(z) d z .
$$

Suppose now that $S$ has no branch point in the interior of $\gamma$, then by using the Cauchy theorem we would then conclude, contrarily to the hypothesis, that $\int_{D} f(z) d \mu=0$. Therefore, we must have an even number of branch points inside $D$. Another important result is that the branch point never touches the curve $\gamma$. This can be proved by noting that $\gamma$ is, by hypothesis, a simple analytic polynomial curve and the Schwarz function $S$ must be analytic on $\gamma$ and on its neighborhood (see $[16,20]$ ), what excludes the branch point touching the curve $\gamma$, concluding the proof.

\section{Conformal Deformation}

In this section we conclude the proof of our main result, Theorem 1. Since the Riemann map $h(z ; s)$ is conformal from the exterior of the unit disk onto the exterior of $\gamma(s)$ it has a well defined inverse from the exterior of $\gamma(s)$ onto the exterior of the unit disk. We shall denote its inverse by $H(z ; s)$ :

$$
h(H(z ; s) ; s)=z
$$

for all $z$ in the exterior of $\gamma(s)$ and $s \in(0,1]$.

The Schwarz functions $S$ can be related to the Riemann map $h$ and its inverse $H$ by the following:

Proposition 5 Let $\gamma$ be a polynomial curve parameterized by $h$. Then the Schwarz function is a biholomorphic map in a neighborhood of $\gamma$ and is given by

$$
S(z)=\bar{h}\left(\frac{1}{H(z)}\right)
$$

where $\bar{h}(w)=r w+\bar{a}_{0}+\bar{a}_{1} w^{-1}+\cdots+\bar{a}_{n} w^{-n}$.

The proof can be found in Refs. [16, 20].

Proposition 6 If $h(z ; s)=r z+\sum_{j=0}^{n} a_{j}(s) z^{-j}$ with the $\alpha_{j}(s)=r^{-j} a_{j}(s)$ satisfying the conditions $(i-i i i)$ of Remark 3, then the inverse function $H(z ; s)$ of $h(z ; s)$ reads

$$
H(z ; s)=\frac{z-a_{0}(s)+\sqrt{\left(z-a_{0}(s)\right)^{2}-4 r a_{1}(s)}}{2 r}+o(s)
$$

for $0<s<\varepsilon$ and $\varepsilon$ small enough. 
Proof: We have by Eqs. (24) and (52)

$$
h(H(z) ; s)=r H(z ; s)+\sum_{j=0}^{n} a_{j}(s) H(z ; s)^{-j}=z
$$

which yields

$$
r H^{2}(z ; s)-\left(z-a_{0}(s)+o(s)\right) H(z ; s)+a_{1}(s)=0 .
$$

Solving the equation for $H$ the result follows. Note that $|H|=\mathcal{O}(1)$ in the neighborhood of $\gamma(s)$.

Remark 8 Equation (53) has branches, namely the minus square root, which can be directly seen from Eq. (54). We do not consider the minus square root, because it does not yield $S(z)=\bar{z}$ on $\gamma(s)$.

Lemma 1 Let $d \hat{\mu}(z ; s)$ be the balayage measure supported on $\gamma(s)$ of the Elbau-Felder ensemble. Assume that the conditions $(i-i i i)$ of Remark 3 hold. Then, given $\varepsilon$ sufficiently small, for $0<s<\varepsilon$ and $f \in \mathcal{H}(D(s))$, we have

$$
\oint_{\gamma(s)} f(z) d \hat{\mu}(z ; s)=\frac{1}{2 \pi r^{2}} \int_{-2 r}^{2 r} f(x) \sqrt{4 r^{2}-x^{2}} d x+o(1) .
$$

Proof. Given $z$ in a neighborhood of $\gamma(s)$ and $\varepsilon>0$ sufficiently small, the Schwarz function of $\gamma(s)$ for $0<s<\varepsilon$ by Proposition 5 reads

$$
S(z ; s)=r \frac{1}{H(z ; s)}+\bar{a}_{0}(s)+\bar{a}_{1}(s) H(z ; s)+\cdots+\bar{a}_{n}(s) H^{n}(z ; s)
$$

which together with (53), yields

$$
S(z ; s)=E\left(z-a_{0}(s)\right)+\Lambda \sqrt{\left(z-a_{0}(s)\right)^{2}-4 r a_{1}(s)}+g(z ; s)
$$

where $E=\left(r^{2}+\left|a_{1}^{2}(s)\right|\right) / 2 r a_{1}(s)$ and $\Lambda=\left(\left|a_{1}^{2}(s)\right|-r^{2}\right) / 2 r a_{1}(s)$, and $g(z ; s)$ is $o(s)$. Introducing

$$
I(s)=\oint_{\gamma(s)} f(z) d \hat{\mu}(z ; s),
$$

by (51), the contour integral will be performed taking into account 56 . The constant and linear term in (56) will give no contribution by Cauchy theorem. Now we need to estimate term depending on $g$. Since $g$ is analytic in a neighborhood of $\gamma(s)$, we have the bound

$$
\frac{1}{2 \pi t_{0}(s)}\left|\oint_{\gamma(s)} g(z ; s) d z\right| \leq \frac{\max _{z \in \gamma(s)}|g(z ; s)|}{2 \pi t_{0}(s)} \oint_{\gamma(s)} d z
$$

Since arc length $\oint_{\gamma(s)} d z$ is finite, $\max _{z \in \gamma(s)}|g(z ; s)|=o(s)$, and by (18) and conditions (ii) of Remark 3.

$$
t_{0}(s)=r^{2}-\left|a_{1}(s)\right|^{2}+o\left(s^{2}\right)=r^{2} s+o(s),
$$


we have that the r.h.s. of $(57)$ is $o(s) / t_{0}(s)=o(1)$. Hence, $\Lambda / t_{0}(s)=-1 / 2 r^{2}+\mathcal{O}(s)$. Also, for $z \in \gamma(s)$,

$$
\frac{\sqrt{4 r a_{1}(s)-\left(z-a_{0}(s)\right)^{2}}}{r a_{1}(s)}=\frac{\sqrt{4 r^{2}-\left(z-a_{0}(s)\right)^{2}}}{r^{2}}+\mathcal{O}(s) .
$$

Combining the estimates, we have

$$
I(s)=\frac{-1}{4 \pi} \oint_{\gamma(s)} f(z) \frac{\sqrt{4 r^{2}-z^{2}}}{r^{2}} d z+o(1)
$$

By using Proposition 2, $\gamma(s)$ may be deformed until it coincides with the branch cut of the square root. Because of the branch, the integrand around the cut will change its sign: for $\gamma$ running from $2 r$ to $-2 r$, the square root becomes $\sqrt{4 r^{2}-x^{2}}$, while running from $-2 r$ to $2 r$ the square root becomes $-\sqrt{4 r^{2}-x^{2}}$. This leads to

$$
I(s)=\frac{1}{2 \pi r^{2}} \int_{-2 r}^{2 r} f(x) \sqrt{4 r^{2}-x^{2}} d x+o(1)
$$

concluding the proof.

\subsection{Proof of Theorem 1:}

We shall prove the item 1). From the initial ensemble with analytic polynomial curve $\gamma$, we have constructed a one parameter a family of curves $\gamma(s, r, \boldsymbol{\tau})$ parameterized by $h(w ; s, r, \boldsymbol{\tau})$ with $|w|=1$ and $s \in\left(0, s_{0}\right]$. We have shown, in Proposition 1 , that under the hypothesis $(25)$ the family $\gamma(s, r, \boldsymbol{\tau})$ is composed of simple closed analytic polynomial curves of degree $n$. It follows from Proposition 3 that for $r$ sufficiently small the hypothesis (25) is fulfilled (see Eq. (48) along with Remark 7). Therefore, $\gamma(s, r, \boldsymbol{\tau})$ is a simple analytic polynomial curve for every $s \in\left(0, s_{0}\right]$. Moreover, $h(w ; s, r, \boldsymbol{\tau})$ acts as the Riemann map from the exterior of the unity circle onto the exterior of $\gamma(s, r, \boldsymbol{\tau})$ for $s \in\left(0, s_{0}\right]$.

The uniqueness of the curve and the fact that the parameters of $h(w ; s, r, \boldsymbol{\tau})$ can be uniquely determined by $t_{0}$ and $\boldsymbol{t}(s)$ follows from the fact that the problem of determining the exterior moments $t_{j}$ out of simple closed analytic polynomial curves has a unique solution also when $t_{2}$ tends to $1 / 2$. The proof of this fact is presented in Proposition 3 . The first part of item 2) is proven in Subsection 4.2 for $t_{0}$ small enough.

We prove the second part of item 2) with help of the balayage techniques. The balayage enables us to sweep all the eigenvalues to the boundary $\gamma(s, r, \boldsymbol{\tau})$ and to analyze the deformation only focusing on $\gamma(s, r, \boldsymbol{\tau})$. In Theorem 4 we deduce an explicit equation relating the balayage measure with the potential $V$ for test function space $\mathcal{H}$. It turns out that the balayage measure is proportional to the Schwarz function.

In Proposition 4 we show that the Schwarz function of every simple closed polynomial curve must have a branch cut which lies in the interior of the curve and the branch points never touch the curve itself. The limit $s \rightarrow 0$, the curve $\gamma(s, r, \boldsymbol{\tau})$ 
collapses into the line, see Proposition 1. In Lemma 1 we control the convergence of the equilibrium measure as $s \rightarrow 0$, Here the converge rate $\Delta_{j}>1$ play a role in controlling the contributions to the Schwarz function is the curve $\gamma(s, r, \boldsymbol{\tau})$ collapses to the support $[-2 r, 2 r]$. Lemma 1 then shows that the equilibrium measures weakly converges to the semicircle law. This concludes the proof.

\section{Examples}

\subsection{Potential of degree 2}

Taking $n=1$ in (6), the potential $V$ reads

$$
V(z)=\frac{z \bar{z}-t_{2} z^{2}-\bar{t}_{2} \bar{z}^{2}}{t_{0}}
$$

Consider the one parameter family for Riemann maps $h(w ; s)=r w+a_{1}(s) w^{-1}$ in which, without loss of generality, $r>0$ and $a_{1}(s)=r(1-s)$ for $s \in(0,1]$. Note that this parameterization fulfills the conditions $(i-i i i)$ by taking $s \mapsto s / 2$. This yields $h(w ; s)=r\left(w+(1-s) w^{-1}\right) \quad t_{0}=r^{2}\left(2 s-s^{2}\right)$ and $2 t_{2}=1-s$, by relations (18) and (19). The support of the eigenvalues is then given by

$$
D(s)=\left\{x+i y \in \mathbb{C}: \frac{x^{2}}{r^{2}(2-s)^{2}}+\frac{y^{2}}{r^{2} s^{2}} \leq 1\right\} .
$$

with major and minor semi-axis given, respectively, by $(2-s)$ and $s$. For every $s \in(0,1]$ the Elbau-Felder conditions are satisfied, namely, $t_{1}(s)=0$ and $\left|t_{2}(s)\right|<1 / 2$. Condition (25): $\xi(s)=r-a_{2}(s)=s>0$ for $s \in(0,1]$, holds and conditions $(i-i i i)$ are trivially satisfied.

The equilibrium measure associated to this potential is uniform within the support

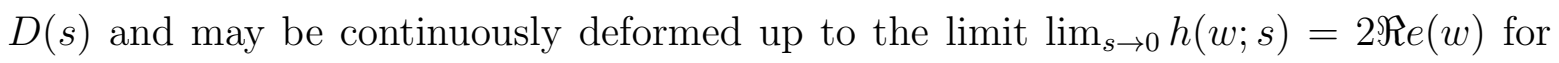
$|w|=1$, in which its boundary becomes the line segment $[-2 r, 2 r]$.

To show that the balayage measure converges to the Wigner measure, we consider the Schwarz function, which can be computed using the formulae in Ref. [20] or using $H(w ; s)$ together with Proposition 5. By equation (51) and the Cauchy theorem, only the non-holomorphic part of the Schwarz function gives a contribution to the integrals. For test functions $f \in \mathcal{H}(D(s))$ and the properties of the balayage measure (23), we have

$$
\int_{D(s)} f(z) d \mu(z ; s)=\frac{1}{2 \pi r^{2}(1-s)} \int_{-2 r \sqrt{1-s}}^{2 r \sqrt{1-s}} f(x) \sqrt{4 r^{2}(1-s)-x^{2}} d x
$$

\subsection{Potentials of Degree 3: Breaking Down the Hypotheses}

If we break the conditions $(i-i i i)$ of Remark $3\left(\Delta_{j}>1\right)$, then it is impossible to deform the equilibrium measure keeping simultaneously the regularity of the polynomial curve 
and a non trivial support. We shall illustrate this scenario for $n=2$ taking $t_{1}=t_{2}=0$. The potential then reads

$$
V(z)=\frac{z \bar{z}-t_{3} z^{3}-\bar{t}_{3} \bar{z}^{3}}{t_{0}} .
$$

By (18) we have $t_{0}(s)=r^{2}-2\left|a_{2}(s)\right|^{2}$ and $3 t_{3}(s)=a_{2}(s) / r^{2}$. The Riemann map is written as $h(w ; s)=r w+a_{2}(s) w^{-2}$. Note that $a_{1}=0$ violates condition $(i i)$. Therefore, the deformation of $\gamma(s, r)$ to a segment of the real line is impossible. The regularity of $\gamma(s, r)$, which is guaranteed by condition (25), requires $\left|a_{2}\right|<r / 2$, while the positive area condition on $t_{0}$ requires $\left|a_{2}\right|<r / \sqrt{2}$.

There is no parameterization that keeps the regularity of $\gamma(s, r)$. When the area $\pi t_{0}$ of $D(s, r)$ converges to zero $\left(a_{2} \rightarrow r / 2\right), \gamma(s, r)$ develops cusps and is no longer regular. Keeping the regularity of the curve, the limit $t_{0} \rightarrow 0$ is possible only taking $r \rightarrow 0$. Therefore, as the area $\pi t_{0}$ converges to zero, hence, $\gamma(s, r)$ collapses to a point.

\section{Conclusion}

We study the conformal deformations of equilibrium measures of Elbau-Felder ensembles to the real line. Special attention is paid to Wigner measures, for which the density of eigenvalues follows the semicircular law. Two ingredients are used to prove the main result. We use bifurcation theory from a single eigenvalue for extending the moment problem to near slit domains, and the concept of balayage measure together with the Schwarz function for assisting the convergence to Wigner measures.

Our approach to the problem considers a family of normal ensembles parameterized by $s$. For each $s \in(0,1]$ fixed the Elbau-Felder results guarantee that the probability of finding eigenvalues outside the curve $\gamma(s, r, \boldsymbol{\tau})$ decays is exponentially fast with $N$. The problem then turns to whether convergence of the eigenvalue density to the equilibrium measure would be uniform as a function $s$. The uniformity of the limit remains an open question.

The result presented in Theorem 1 is in a way universal, in the sense that it does not depend on the initial ensemble one starts with. It would be interesting to analyze to deformation of the Elbau-Felder ensembles to the real line without the assumption on the convergence rate $-\Delta_{j}>1$ in item 3) of Theorem 1 . Here the resulting equilibrium measure, if well defined, might deviate from the semicircular law, and it might also depend on the initial ensemble under consideration.

We would like to acknowledge the financial of the Brazilian agencies FAPESP under grant 07/04579-2 (TP), CNPq under 151976/2007-4 (AMV) and 474647/2009-9 (TP). DHUM is partially supported by CNPq. We would like also to thank the anonymous referees for valuable comments and suggestions. 


\section{Appendix A. Solution of (33)}

Lemma 2 The linear system of equations (33) has a unique solution $\boldsymbol{\varphi}=T \boldsymbol{v}+B \overline{\boldsymbol{v}}$ where $B=\left(1-|k|^{2}\right)^{-1} J K$ with $k=K_{11}$ and $T=J+\bar{k} B$, provided $|k| \neq 1$.

Proof. Equation (33) is solvable if and only if

$$
\left(\begin{array}{cc}
B & T \\
\bar{T} & \bar{B}
\end{array}\right)\left(\begin{array}{cc}
-\bar{K} & J^{-1} \\
J^{-1} & -K
\end{array}\right)=\left(\begin{array}{ll}
I & 0 \\
0 & I
\end{array}\right)
$$

holds for some $(n+1) \times(n+1)$ complex matrices $B$ and $T$ and this is equivalent to

$$
\begin{aligned}
& -B \bar{K}+T J^{-1}=I \\
& B J^{-1}-T K=0 .
\end{aligned}
$$

Let us assume that $B$ has its first column given by $\boldsymbol{b}$ and 0 everywhere else (so $B$ has the same form of $K)$ and let $T=J+\bar{k} B$. Note that $K A=a_{11} K\left(B A=a_{11} B\right)$ holds for any matrix $A=\left[a_{i j}\right]$, such that $a_{i j}=0$ for $j>1$. Substituting $T$ in (A.1), we have

$$
\begin{aligned}
B J^{-1}-T K & =B J^{-1}-(J+\bar{k} B) K \\
& =B-J K-|k|^{2} B=0
\end{aligned}
$$

which implies

$$
\begin{aligned}
B & =\frac{1}{1-|k|^{2}} J K \\
T & =J+\frac{\bar{k}}{1-|k|^{2}} J K .
\end{aligned}
$$

and concludes the proof of the lemma. The uniqueness follows by linearity.

[1] M. L. Mehta, Random Matrices Academic Press, San Diego, CA (1991)

[2] P. Deift. "Orthogonal Polynomials and Random Matrices: A Riemann-Hilbert Approach". New York University, New York. Lecture Notes 3, AMS (2000)

[3] K.B. Efetov, Phys. Rev. Lett. 79, 491 (1997)

[4] N. Hatano and D. R. Nelson, Phys. Rev. Lett. 77, 570 (1996)

[5] V.V. Sokolov and V.G. Zelevinsky, Nucl. Phys. A 504, 562 (1989); F. Haake, F. Izrailev, N. Lehmann, D. Saher and H.J. Sommers, Z. Phys. B 88, 359 (1992); M. Müller, F.M. Dittes, W. Iskra, and I. Rotter, Phys. Rev. E 52, 5961 (1995)

[6] J.J. M. Verbaarschot and I. Zahed, Phys. Rev. Lett. 70, 3852 (1992), G. Akemann, J. Phys. A: Math Gen. 36, 3363 (2003)

[7] Y. V. Fyodorov and H.J. Sommers, Jour. Phys. A: Math. Gen. 36, 3303 (2003).

[8] L.L. Chau and Y. Yu, Phys. Lett. A 167, 452 (1992)

[9] H. Hedenmalm and N. Makarov, Quantum Hele-Shaw flow, arXiv: math. PR/0411437

[10] P. B. Wiegmann, A. Zabrodin, Comm. Math. Phys. 213, 523 (2000)

[11] I.K. Kostov, I. Krichever, M. Mineev-Weinstein, P.B. Wiegmann, A Zabrodin, Math. Sci. Res. Ins. Publ. 40, 285 (2001)

[12] A. Marshakov, P. Wiegmann, A. Zabrodin, Commun. Math. Phys. 227, 131 (2002)

[13] Y. V. Fyodorov, B. A. Khoruzhenko, H.-J. Sommers, Phys. Rev. Lett. 79(4), 557-560, (1997). 
[14] A. Jarosz and M.A. Nowak, J. Phys. A: Math. Gen. 39, 10107 (2006).

[15] I. Krichever, A. Marshakov, A. Zabrodin, hep-th/0309010 (2003)

[16] P. Elbau; G. Felder, Comm. Math. Phys. 259, 433 (2005)

[17] M. G. Crandall, "An Introduction to Constructive Aspects of Bifurcation and the Implicit Function Theorem" in Application of Bifurcation Theory, ed. Rabinowitz. Academic Press (1977)

[18] H. S. Shapiro, "The Schwarz function and its generalization to higher dimensions". John Willey \& Sons (1992)

[19] E. B. Saff and V. Totik. "Logarithmic potentials with external fields". Springer, New York-Berlin (1997)

[20] P. J. Davis. "The Schwarz functions and its Applications". The Carus Mathematical Monographs $\mathrm{n}^{\circ}$ 17. The mathematical association of America. 